\title{
Capecitabine/Oxaliplatin/Trastuzumab Regimen
}

National Cancer Institute

\section{Source}

National Cancer Institute. Capecitabine/Oxaliplatin/Trastuzumab Regimen. NCI

Thesaurus. Code C160542.

A regimen consisting of capecitabine, oxaliplatin, and trastuzumab that may be used in the treatment of human epidermal growth factor receptor 2 (HER2)-positive advanced gastric cancers. 